\title{
Research Paper \\ Prediction of the Anxiety Sensitivity Based on Lifestyle Features in Students of Qazvin University of Medical Sciences
}

\author{
*Ahmad Poursharifi ${ }^{1}\left(\mathbb{C}\right.$, Asghar Jafari $^{2}$ (1)
}

1. Department of Counseling, Faculty of Humanities, Abhar Branch, Islamic Azad University, Abhar, Iran.

2. Department of Psychology, Faculty of Humanities, University of Kashan, Kashan, Iran.

\begin{tabular}{c|c|c|}
\hline $\begin{array}{l}\text { Use your device to scan } \\
\text { and read the article online }\end{array}$ \\
Citation Poursharifi A, Jafari A. Prediction of the Anxiety Sensitivity Based on Lifestyle Features in Students of Qazvin University of Med- \\
ical Sciences. The Journal of Qazvin University of Medical Sciences. 2019; 23(2):152-163. https://doi.org/10.32598/JQUMS.23.2.152
\end{tabular}

Received: 08 Aug 2018 Accepted: 24 Nov 2018 Available Online: 01 Apr 2019

\section{AB STRACT}

Background Anxiety sensitivity is the fear of anxiety and anxiety-related symptoms that may not only affect lifestyle but also result in physical and psychological damages.

Objective The present study aimed to predict anxiety sensitivity based on the components of lifestyle in the students of Qazvin University of Medical Sciences.

Methods This study used a descriptive correlational design. The study population consisted of all students (2260 numbers) of undergraduate, postgraduate, and medical students of Qazvin University of Medical Sciences in the second half of the academic year 2016-2017. According to the Morgan Table, 350 samples were selected by simple random sampling method. The study data were collected by questionnaires of anxiety sensitivity and lifestyle and were analyzed by the Pearson coefficient and multiple regression.

Findings In this study, 233 students were female, and 117 were male. Based on the results, there was a significant relationship between the components of lifestyle (belonging-social interest, coping, responsibility, need to approval, cautiousness, supportive) and anxiety sensitivity $(P<0.01)$. Also, lifestyle predicts anxiety sensitivity. The results of multiple regression showed that lifestyle and its components (belonging-social interest, coping, responsibility, need for confirmation, cautiousness, and supportive) positively predict anxiety sensitivity.

Conclusion According to the study results, the anxiety sensitivity is partially predictable based on the components of lifestyle. Therefore, to prevent and decrease the level of anxiety sensitivity in students, we suggested to promote and optimize the components of their lifestyle.

\section{Extended Abstract}

\section{Introduction}

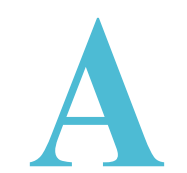

nxiety sensitivity (AS) as a cognitive variable reflects individual differences that are characterized by fear of anxietyrelated sensations and indicates their ten- dency to believe these feelings have some destructive consequences [1]. AS is the fear of anxiety and its symptoms that can affect lifestyle and cause physical and psychological damages. Lifestyle is a reflection of how people live according to their constructs, such as social values, attitudes, and activities that are formed by a combination of behavior and habits throughout life, followed

* Corresponding Author:

Ahmad Poursharifi

Address: Department of Counseling, Faculty of Humanities, Abhar Branch, Islamic Azad University, Abhar, Iran.

Tel: +98 (28) 33341934

E-Mail: a.poorsharifi@gmail.com 
by socialization. The purpose of this study is to evaluate the predictability of AS based on lifestyle components in students of Qazvin University of Medical Sciences.

\section{Materials and Methods}

This research is a descriptive correlational study. The study population consists of all undergraduate, graduate, and postgraduate students $(\mathrm{n}=2260)$ of Qazvin University of Medical Sciences (QUMS). According to Morgan's table, the sample size was determined 350. Considering the possibility of dropout in the samples, 500 questionnaires were distributed among them, and 350 ones were completed and returned. Simple random sampling technique was used for recruiting samples. The inclusion criteria were as follows: having no probation and moral problems, psychosis or neurosis problems; studying in the second semester 2016-2017; and having Iranian nationality. Anxiety Sensitivity Index developed by Reiss et al. [14] was used for measuring students' AS, and Adlerian Lifestyle Questionnaire developed by Adler et al. [17] for assessing their lifestyle quality. For analyzing the collected data, the Pearson correlation test and multiple regression analysis (concurrent approach) were used. The information on students was kept confidential.

\section{Results}

Of 350 participants, 233 were female and 117 male. A total of 55(15.7\%) students were undergraduate, $25(7.1 \%)$ discontinuous undergraduate, $186(53.1 \%)$ graduate, and $84(24 \%)$ postgraduate. Moreover, 44(12.6\%) students were from the School of Medicine, 50(14.3\%) from School of Dentistry, 61(17.4\%) from School of Public Health, 78(22.3\%) from School of Paramedical Sciences, and 117(33.4\%) from School of Nursing and Midwifery. To evaluate the predictability of AS with lifestyle factors, multiple regression analysis (concurrent approach) was carried out, and their correlation was examined using the Pearson correlation test.

The results showed that lifestyle factors, including basic (belonging/social interest, taking charge, going along, want recognition, being cautious) and secondary factors (harshness entitlement, liked by all, striving for perfection, and softness), have a significant correlation with AS ( $\mathrm{P}<0.01)$. Based on regression analysis results, lifestyle and its 5 basic and secondary factors could predict AS.

\section{Conclusion}

The results suggest that changes in AS are partially predictable based on lifestyle factors. Therefore, to prevent and reduce the AS level in students, it is suggested that their lifestyle be promoted and modified. In a study on the effect of lifestyle modifications on the prevention of common mental disorders, including depression, it was reported that psychosocial factors play an important role in causing mental disorders [19]. This result is consistent with the findings of other studies. Alimehdi et al. reported the effectiveness of cognitive-behavioral therapy and mindfulness-based stress reduction on AS [21]. There are some studies whose results are against our result.

For example, Saedi et al. studied the relationship among quality of life, lifestyle, academic performance, and academic achievement in university students and reported that among lifestyle factors, "belonging/ social interest", "want recognition" and "being cautious" could predict academic achievement [7]. However, quality of life, lifestyle, and the academic performance had no association with academic achievement. The results in the present study revealed that the basic (belonging/social interest, taking charge, going along, want recognition, being cautious) and secondary factors (harshness entitlement, liked by all, striving for perfection, and softness) of lifestyle had a significant association with AS, and they could positively predict it.

\section{Ethical Considerations}

\section{Compliance with ethical guidelines}

This article was approved by the thesis (No. 20721603952048) at the Islamic Azad University of Abhar.

\section{Funding}

This study is based on of the first author Ahmad Poursharifi's Master's thesis in Consulting and Guidance, which was conducted at the Department of Counseling, Faculty of Humanities, Abhar Beranch, Islamic Azad University.

\section{Authors' contributions}

Conceptualization: Asghar Jafari; Research, review, editing, and finalization: Ahmad Poursharifi.

\section{Conflicts of interest}

The authors declared no conflict of interests.

\section{Acknowledgements}

The authors would like to thank the assistant of educational affairs at Qazvin University of Medical Sciences and students for their valuable cooperation. 
This Page Intentionally Left Blank 


\title{
ييشبينى حساسيت اضطرابى بر اساس مؤلفهایى سبك زندتى در دانشجويان دانشعًاه علوم يزشكى قزوين
}

\author{
"احمد يورشريفى'ه، اصغر جعفرى
}

ا - كروه مشاوره، دانشكده علوم انسانى، واحد ابهر، دانشكاه آزاد السلامى، ابهرة، ايران.

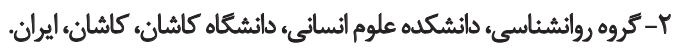

\begin{abstract}
(n)

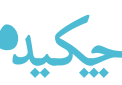

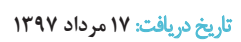

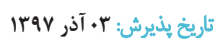
أنمينه حساسيت اضطرابى ترس از اضطراب و نشانههاى آن است كه هم بر سبك زندكى ثأثير بكنارد و هم به آسيبهاى جسمانى و

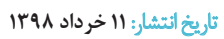

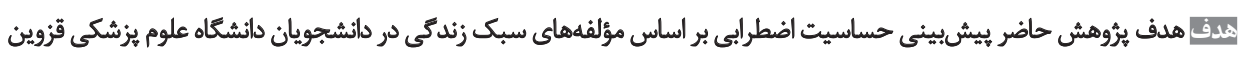

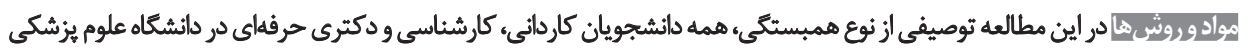

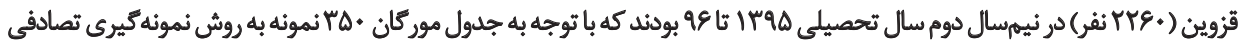

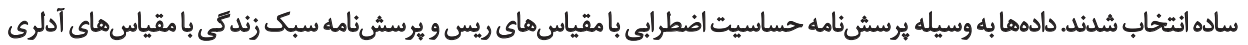

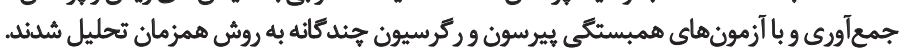

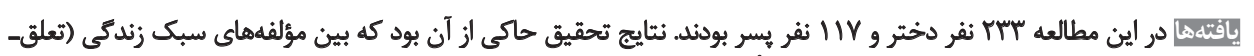

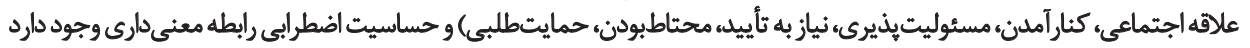

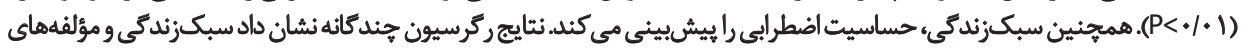

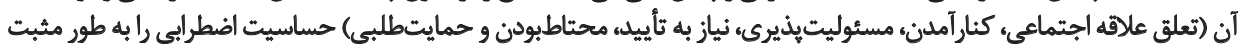

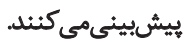

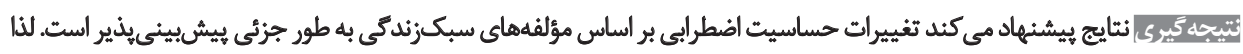

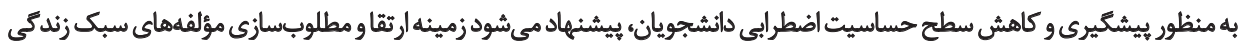

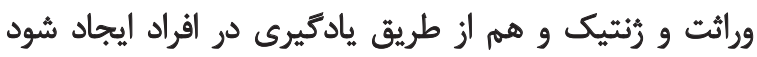

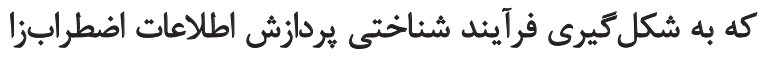

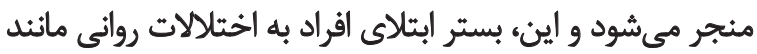

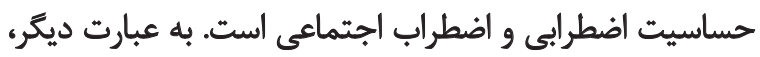

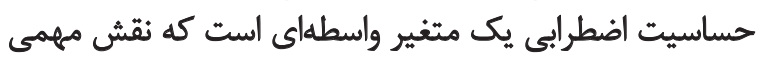

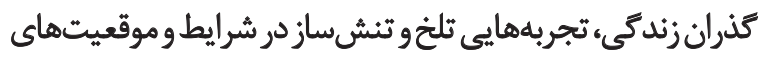

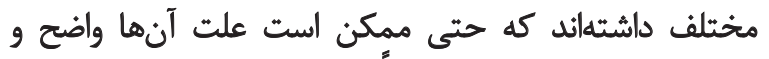

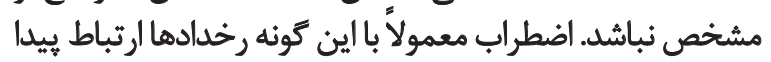

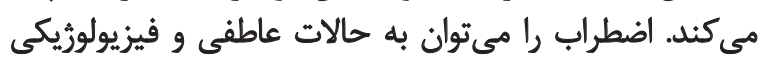

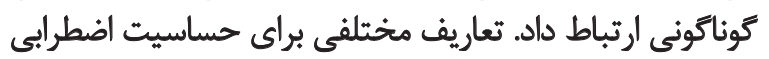

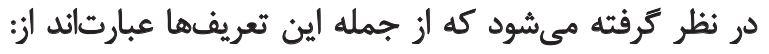

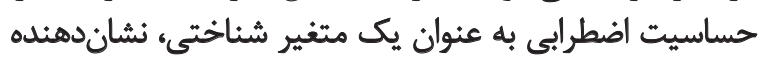


كه اين مطالعات به رابطه سبك زندكى با متغيرهايى مرتبط با

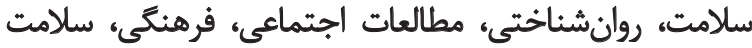

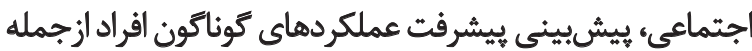

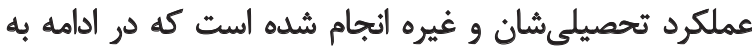

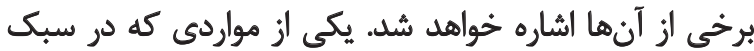

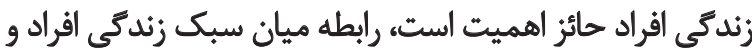

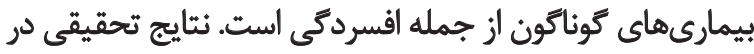

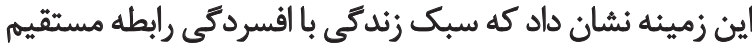

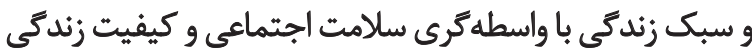

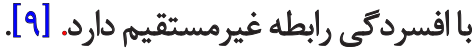

در يك ئروهش، سبك زندكى سلامتمحور به عنوان يديدهاي

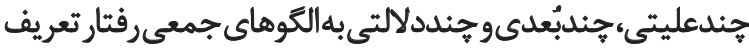

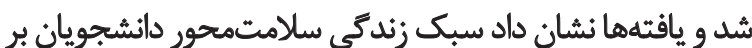
حسب جنسيت، سن، قوميت، وضعيت تأهل و نوع دانشكان انداه آنهان

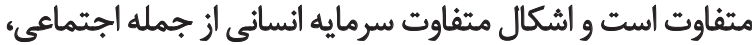

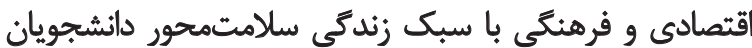

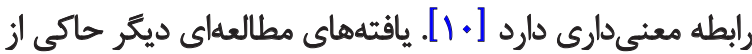

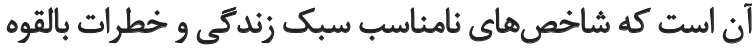

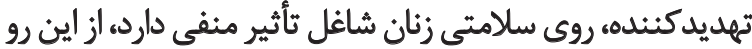

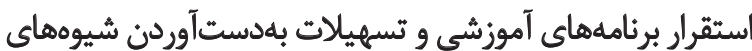

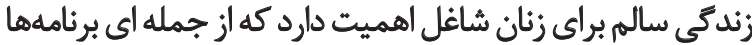

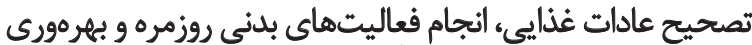

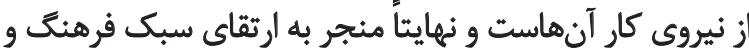

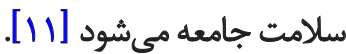

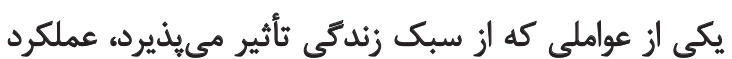

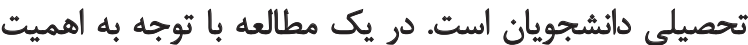

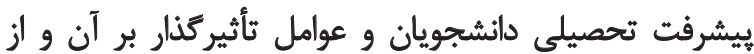

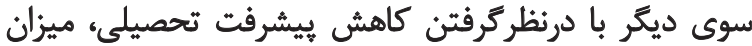

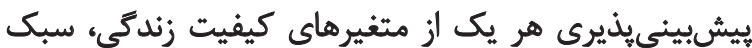

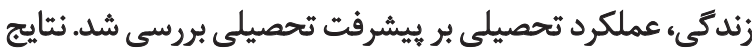

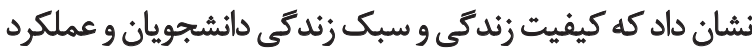

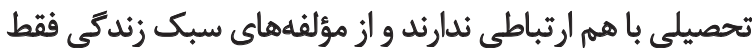

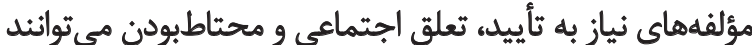

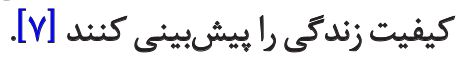

دستهبندى عوامل تأثير كذار در بيشرفت و عملكرد تحصيلى

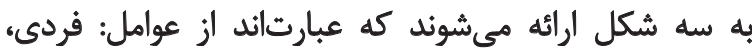

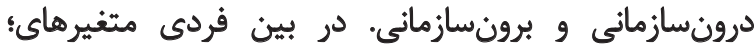

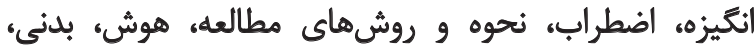

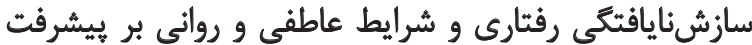

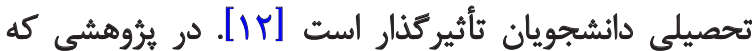

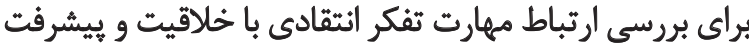

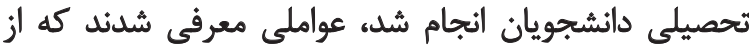
يك سو بر فزونى ثفكر خلاق و خلاقيت اندان دانشجيت معرفيان ثأثير
بين تنشها و بيمارىها دارد كه اين خود تأثير بسزايى درا

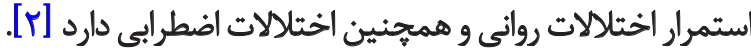

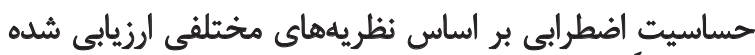

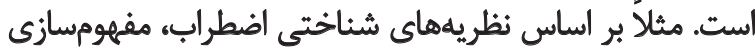

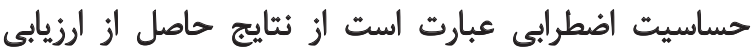

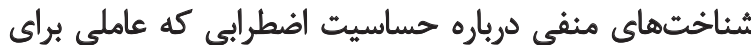
ايجاد خطر و دريتيجه تداوم اضطراب است حسات اضطرابي

مطالعههاى اخير نشان مى دهد حساسيت اضطرابى به عنوان عاملى آسيبيذير به ساير اختلال هائ روانى از جمله إنهائ اختلال

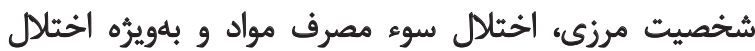

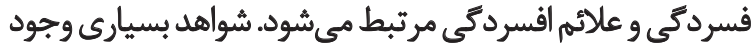

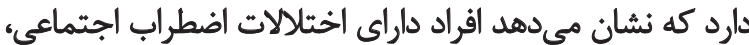

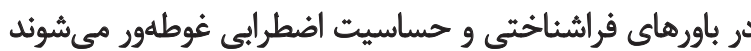

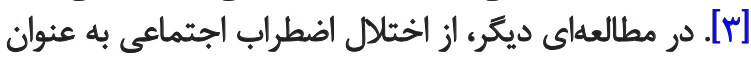

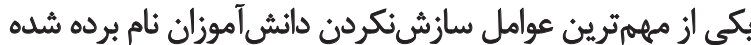

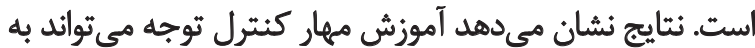

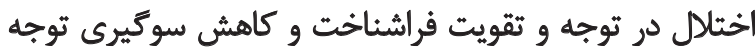

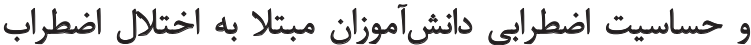

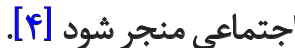
همجينين حساسيت اضطرابى و مهارت تنظيم هيجانى، در

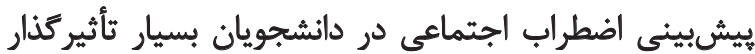

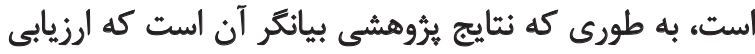

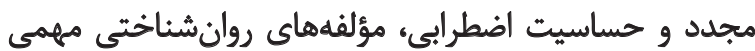

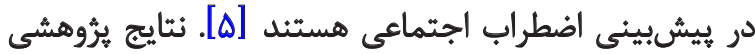

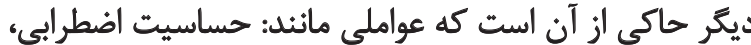

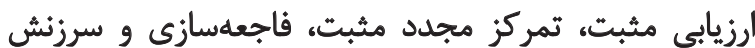

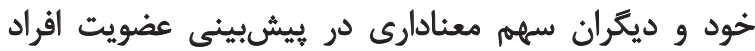

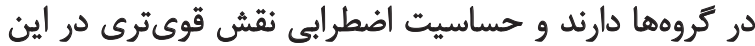

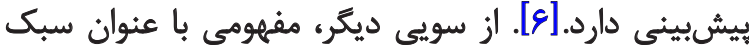

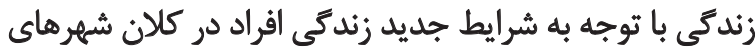

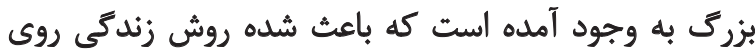

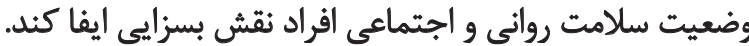

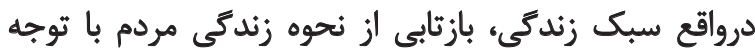

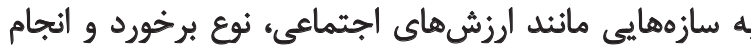

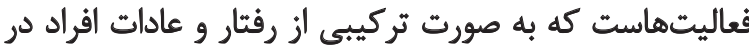

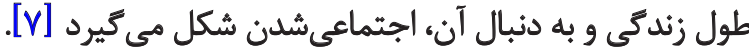

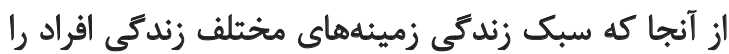

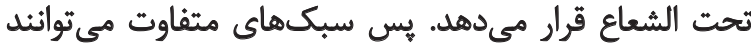

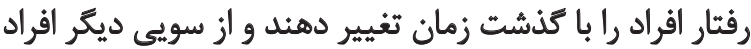

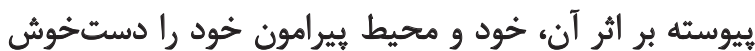
تغيير مى كنيند [1]]. ناكنون مطالعات فراوانى درباره سبك زندكى انجام شده است 
موضوع تحقيق حاضرء از دو برسشنامه حساسيت اضطرابي

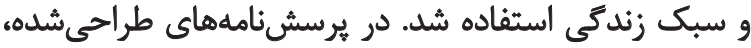

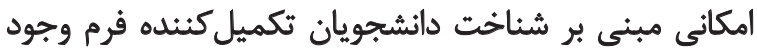

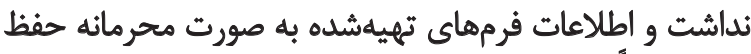
شد و وفاً از نتايج استفاده شد.

يرسشنامه حساسيت اضطرابى با مقياسهاى ريس يك

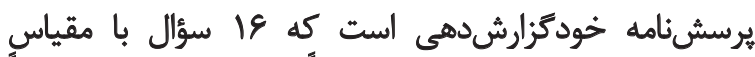

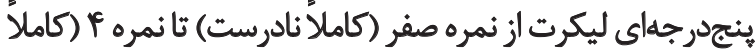

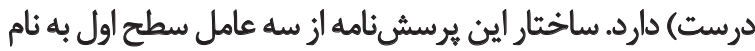

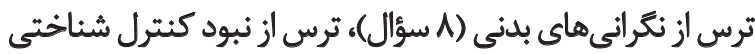

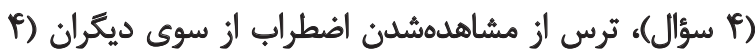

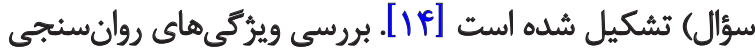

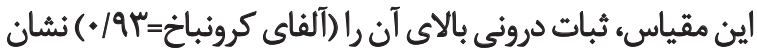

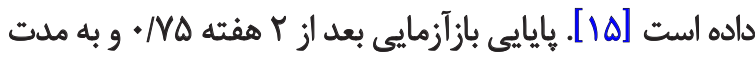

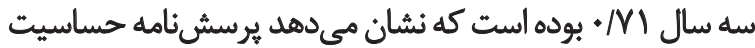

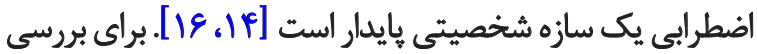

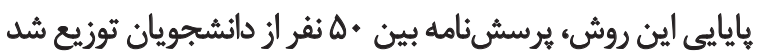

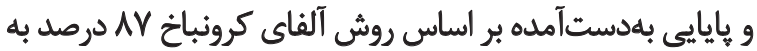

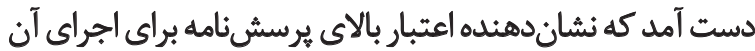
در بروهش حاضر است.

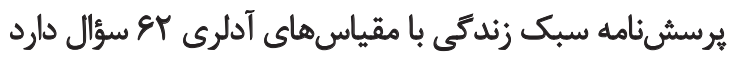

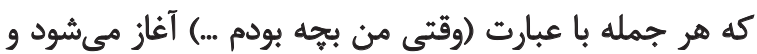

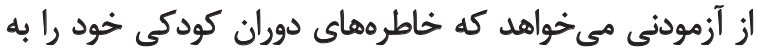

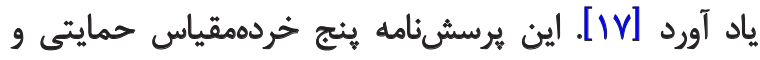

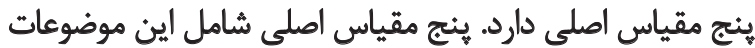

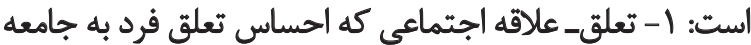

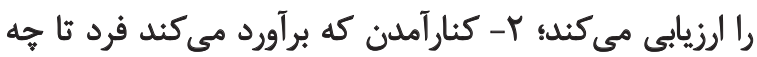

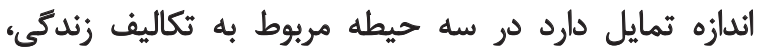

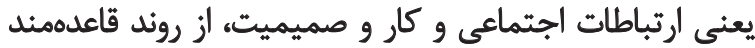

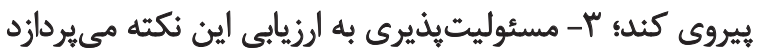

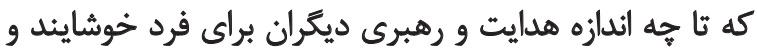

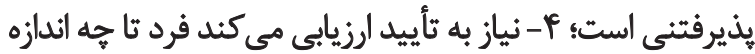

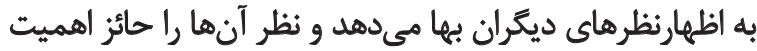

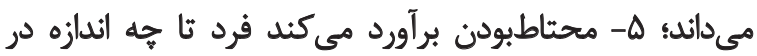
موقعيتهاى بينفردى احساس اعتماد و امنيت مى كنيد.

ينج مقياس حمايتى يرسشنامه نيز به اين معنا است: إ-

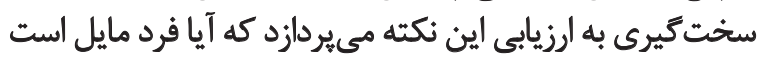

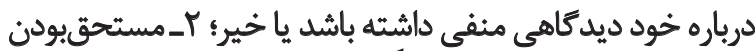

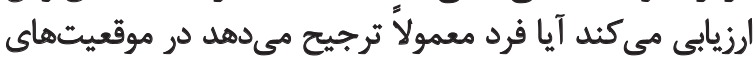

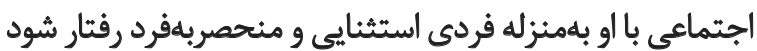

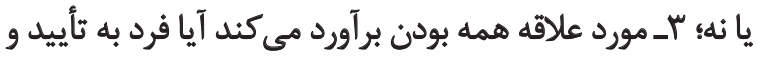

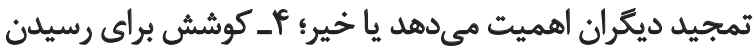

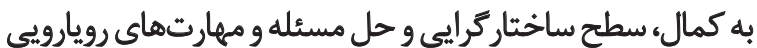

هي كذارند و از سويى ديكر به ارتباط بيشتر دانشعاهها با مراكز

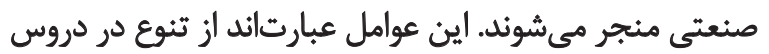

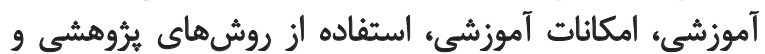

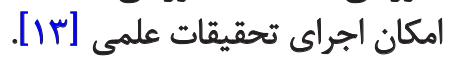

ور فصل امتحانات تغييراتى در وضعيت جسمانى دانش آموزان

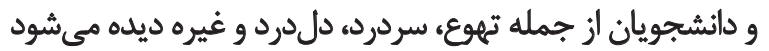

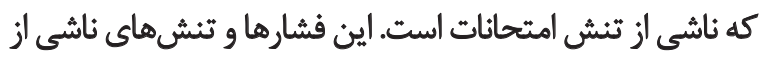

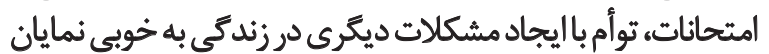

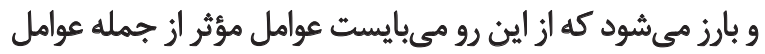

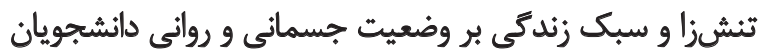

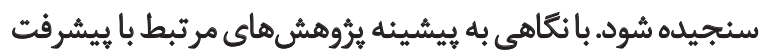

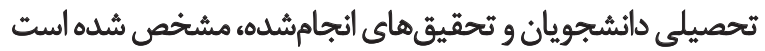

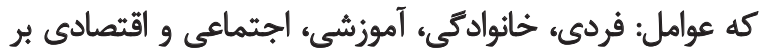

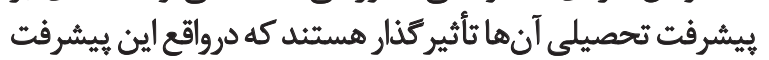

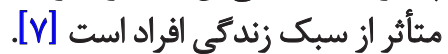

به همين دليل اين مطالعه با هدف بررسى بِيشبينى

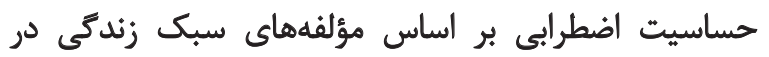

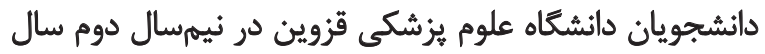
تحصيلى وq-ه انجام شد.

\section{مواد وروشها}

اين مطالعه توصيفى از نوع همبستكى است كه جامعانه آمارى

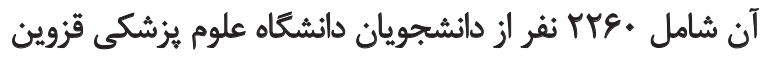

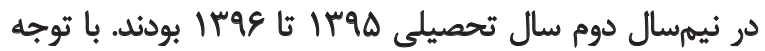

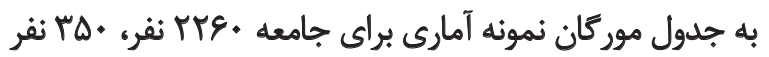

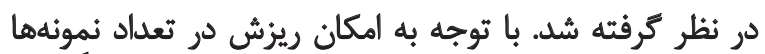

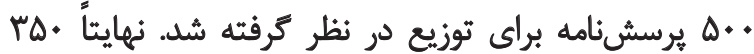

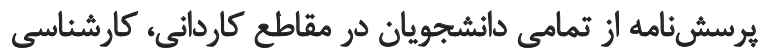

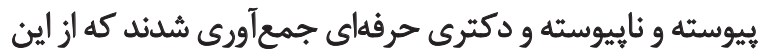

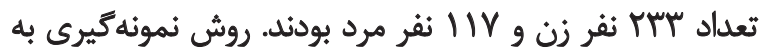
صورت تصادفى ساده بود. ابتدا فهرست آمارى تعداد دان دانشجويان

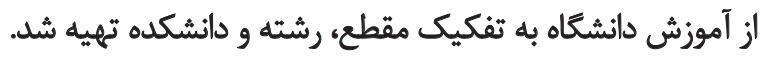

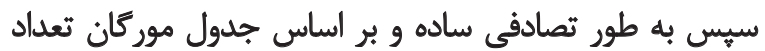

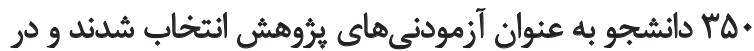

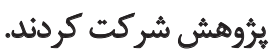

معيارهاى ورود و خروج افراد اين بود كه شركتكننده

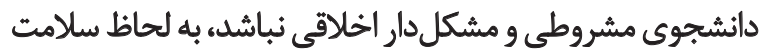

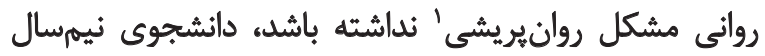

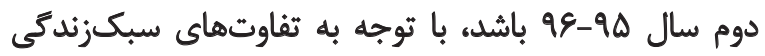

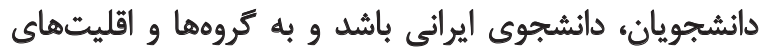

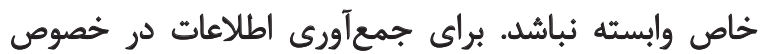

1. Psychosis 
جدول (. شاخص هاى توصيفى مربوط به متغيرهاى مطالعه در كل دانشجويان (• ب نفر)

\begin{tabular}{|c|c|}
\hline ميانكين +انحراف معيار & مثثير \\
\hline WVINE $\pm N T R$ & حساسيت اضطرابى \\
\hline $\mid F T / M T \pm I F / \lambda$ & سبك زندكى \\
\hline$W(1 \pm f|e|$ & نكرانىهايى بدنى \\
\hline VEE $\pm Y / P \Delta$ & نبود كثترل ششاختى \\
\hline 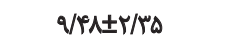 & مشاهده اضطراب توسط ديكران \\
\hline IERADT/AT & تعلت_علاقه اجتماعى \\
\hline TH/TAETIT & كنار آمدن \\
\hline $\mid W T E \pm T / 1$ & مستوليتبذيزي \\
\hline $10 / V q \pm T / R T$ & نياز به تأييد \\
\hline W/FAET/PD & محتاطبودن \\
\hline QVAV $\pm V / F$ & حمايتطلبيى \\
\hline
\end{tabular}

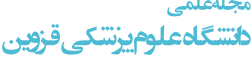

حساسيت اضطرابى (شامل: مهمهبون؛ عصبى به نظر نرسيدن،

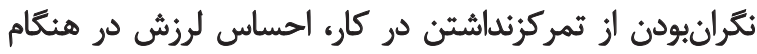

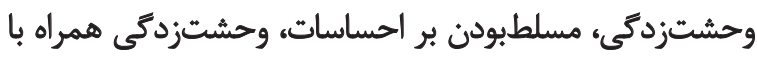

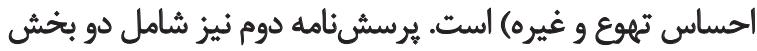

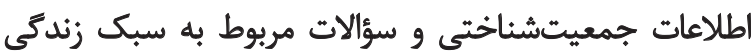

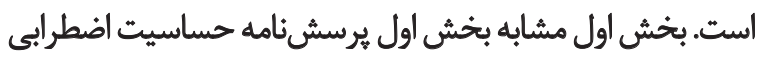

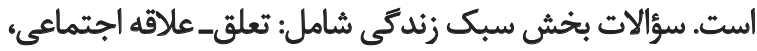

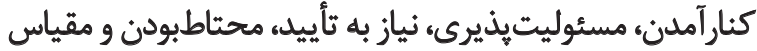

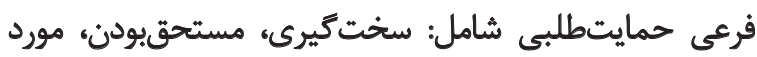
علاقه همه بودن، كوشش براي رسيدن به بمال كمال و ملايمت است. براي توصيف دادهها در سطح آمار توصيفي از فراواني، مجموع،

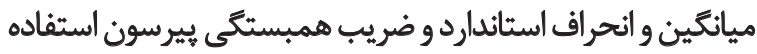

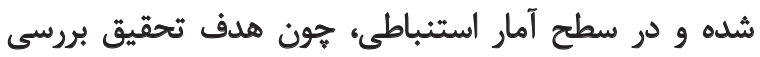

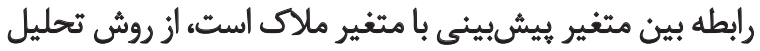

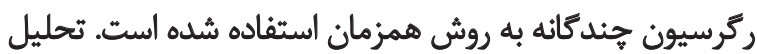

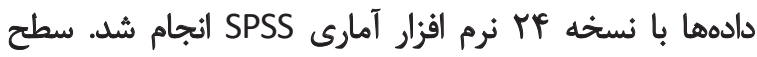

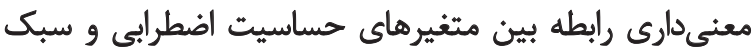

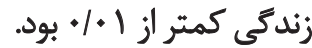

batcollo

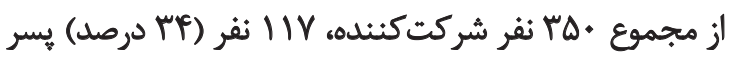

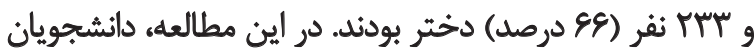

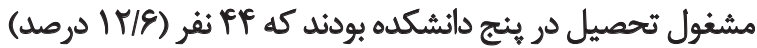

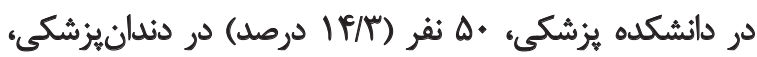

با فشار روانى را در شخص ارزيابى مي كند؛ هــ ملايمت براي

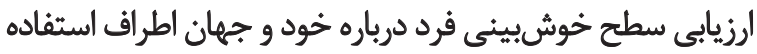

$$
\text { مي شود. }
$$

براى بررسى هايايى و اعتبار يرسشنامه، سازندكان آزمونه

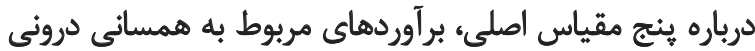

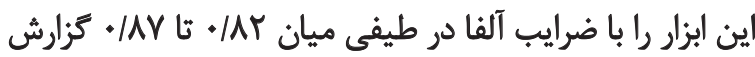

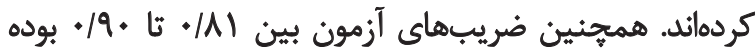

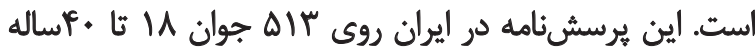

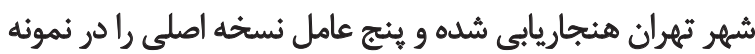

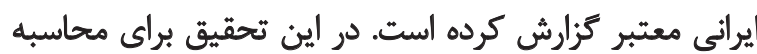

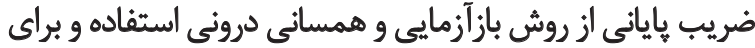

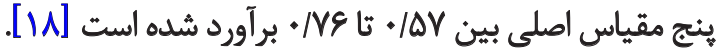
براى بررسي اعتبار برسشئامه از اعتبار صورى (محتوايى)

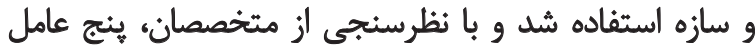

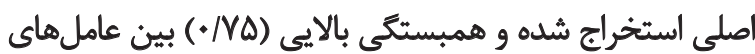

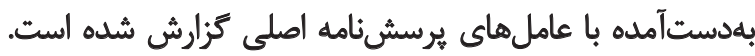

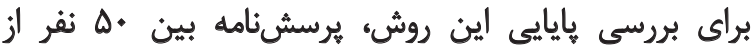

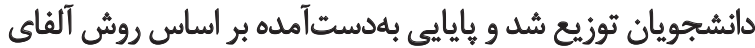

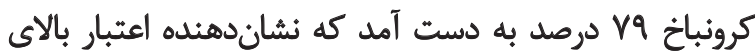

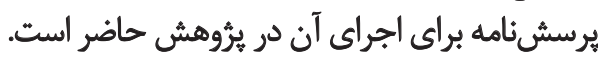
سؤالات يرسشنامه اول عبارت است از دو بخش اطلاعات

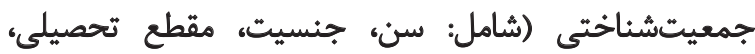

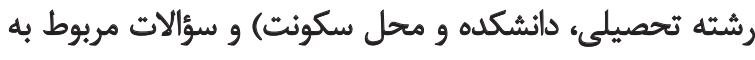


جدول ب. خلاصه مدل بين مؤلفهماي سبك زندكى و حساسيت اضطرابى براساس روش همبستكى بيرسون

\begin{tabular}{|c|c|c|c|c|}
\hline خطاى استاندارد برأورد & ضريب تعيين تعديلشده & ضريب تعيين & همبستكى حندكانه & 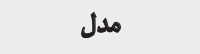 \\
\hline$r / \pi r$ & $\% 1^{\mathrm{a}}$ & H.r & זו/. & تعلق - علاقه اجتماعى \\
\hline P/TA & $.1 . \mathrm{e}^{\mathrm{a}}$ & .1 .8 &.$/ 4 \varphi$ & كثارآملن \\
\hline$r / \mu$ & $\% / \mu^{a}$ &.$/ F \Delta$ &.$/ M$ & مستوليتيذيرى \\
\hline F/TA & $\% \cdot v^{a}$ & $\%$ & 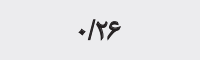 & نياز به تأييد \\
\hline$F / T_{1}$ &.$\% F^{\mathrm{a}}$ & $\% \Delta$ &.$/ r$ & محثاطبودن \\
\hline F/TE &.$/ 9 x^{2}$ & $.18 \Delta$ & . Tra & حمايتطلبي \\
\hline
\end{tabular}

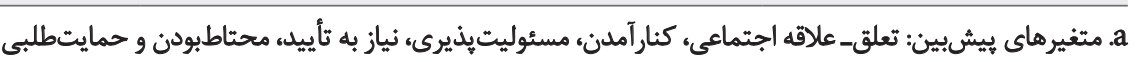

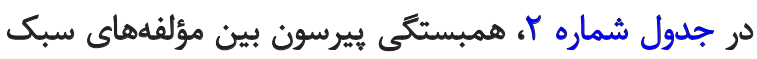

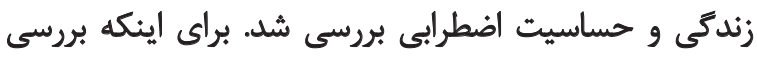

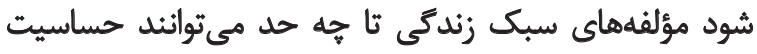

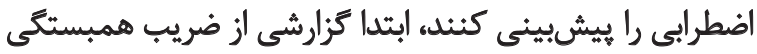

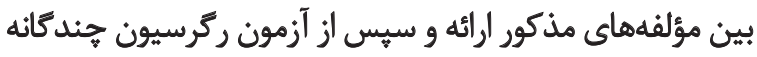

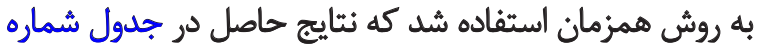
ب ارائه شده است.

با توجه به نتايج بهدستآمده از جدول شماره به از آنجا كه

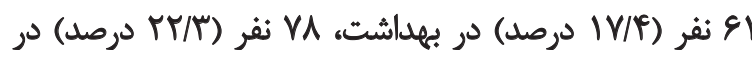

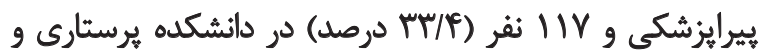
مامايى بودند. نتايج توصيفى نمرههاى سبك زندئى و مؤلفه وهاى

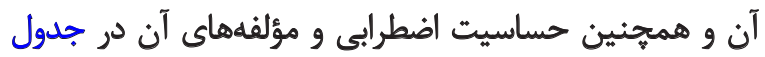

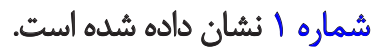
براى بررسى زيشبينى حساسيث اضطرابى با مؤلفه زهاى

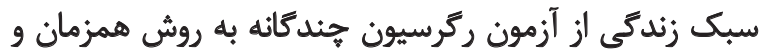

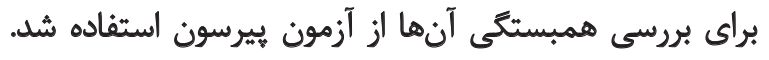

جدول با. آزمون ركر سيون جند كائه به روش همزمان بين مؤلفههاى سبك زئدكى با حساسيت اضطرابى

\begin{tabular}{|c|c|c|c|c|c|}
\hline سطح معني داري & $\beta$ & ميانئين مجذوراث & مجموع مجذورات & منيع تغيير يذيرى & مثغيرهاي رتر سيون \\
\hline$+1+1$ & m & $\begin{array}{l}|M T / T| . \\
\mid V \cdot 9\end{array}$ & 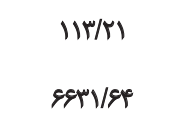 & باقئميون & تعلق_علاقه اجتماعى \\
\hline .1 .01 &.$/ M F$ & $\begin{array}{l}r q+/ r F \\
\text { Wre }\end{array}$ & $\begin{array}{l}r q+/ \pi f \\
g r \Delta f / q 1\end{array}$ & باقى ماندسيون & كثنارآملن \\
\hline.$* 1$ &.$/ M$ & $\begin{array}{l}r+r / P \\
\text { WDI }\end{array}$ & $\begin{array}{l}r+w / s \\
\text { enpl/se }\end{array}$ & ركاقريون & مسئوليتيذيرى \\
\hline$+1++1$ & ( & $\begin{array}{l}\text { Fer/AS } \\
W \cdot \Delta\end{array}$ & $\begin{array}{l}\text { PET/AS } \\
\text { RAA/r }\end{array}$ & 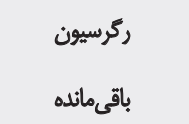 & نياز به تأييد \\
\hline$+1++1$ &.$/ 4$ & $\begin{array}{l}\text { YEV/TA } \\
\text { WEI }\end{array}$ & $\begin{array}{l}\text { rew/Tl } \\
\text { erw/er }\end{array}$ & ركاقريون & هحتاطيونن \\
\hline $.1 . .1$ & . 1 Q & $\begin{array}{l}\text { Pre/TT } \\
\text { WIT }\end{array}$ & $\begin{array}{l}\text { PTE/TT } \\
\text { ET+NOP }\end{array}$ & باقى برسيون & حمايتطلبى \\
\hline
\end{tabular}

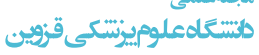




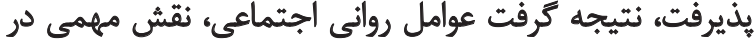

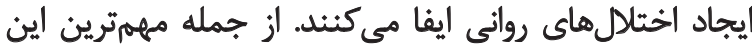

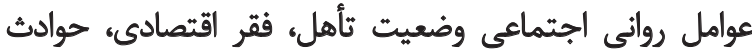

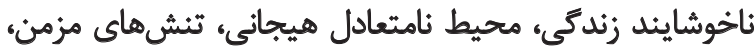

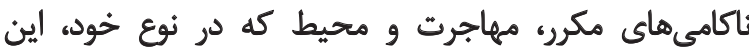

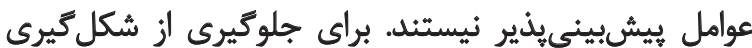

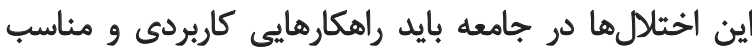

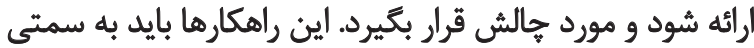

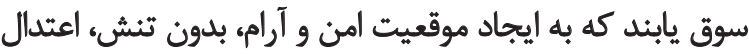

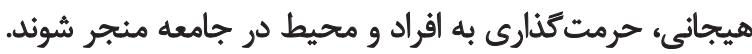

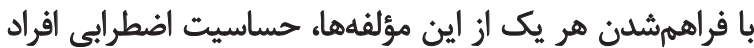

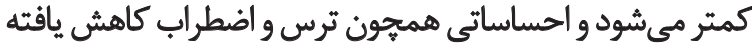

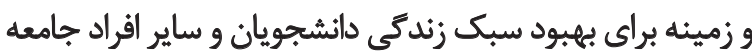

فراهم مىشود [19]

نتايج مطالعهاي با هدف مشخصكردن ارتباط باورهاى

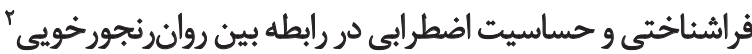

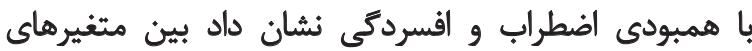
روانرنجورخويى، باورهاى فراشناختى و حساسيت اضطرابي إنى

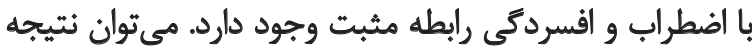

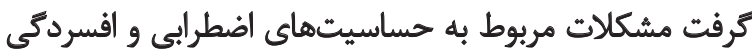

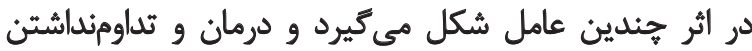

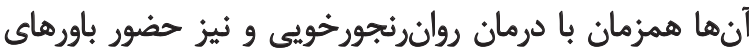

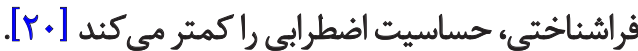

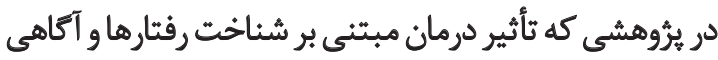

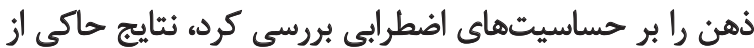

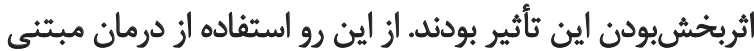

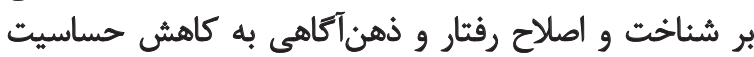

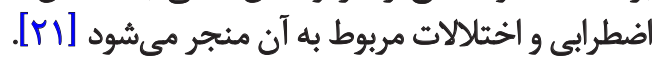
البته نتايج مطالعاتى هم مغاير با مطالعه حاضر بودند، از جمله در مطالعهاى كه رابطه كيفيت، سبك زنائند

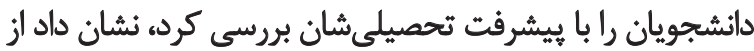

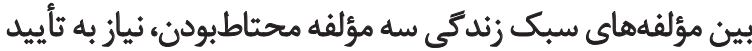

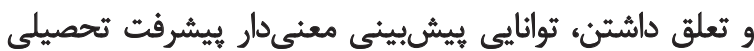

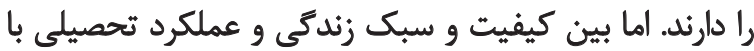

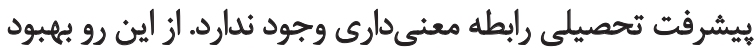

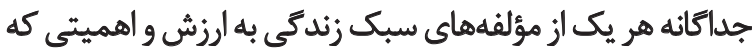

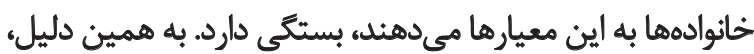

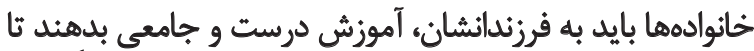

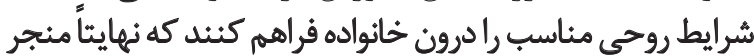
به بيشرفت تحصيلى فرزندان مى دشود در مطالعهاي ديكر كزارش شده است كه هيج يك از مؤلفههاي
سطح معنى دارى مؤلفه تعلقـ علاقه اجتماعى برابر با ب.

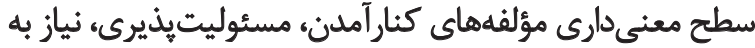

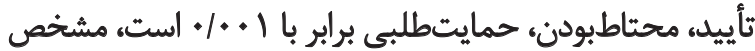

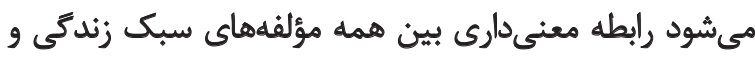
حساسيت اضطرابى وجود دارد.

طبق جدول شماره r تعلق- علاقه اجتماعى، كنارآمدن،

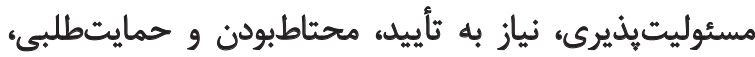

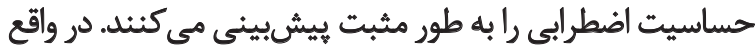

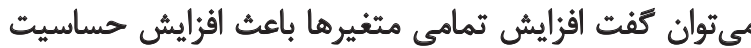

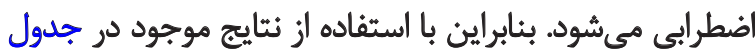

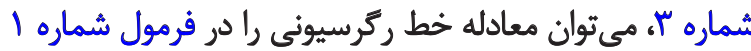

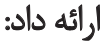

$Y=22 / 17+0 / 09 X_{1}+0 / 4 X_{2}-0 / 03 X_{3}$

$+0 / 23 x_{4}+0 / 06 x_{5}+0 / 1 x_{6}$

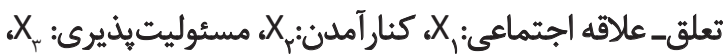

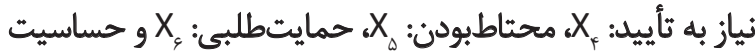

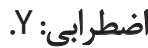

بنابراين با مشاهده معادله خط ركرسيون ميتوان به اين ماين

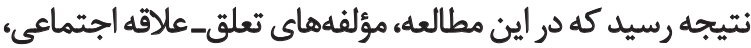

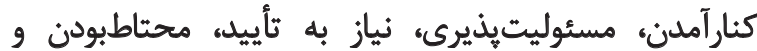

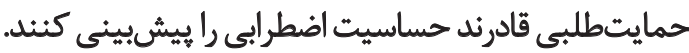

\section{بحث و نتيجها كَيرى}

نتايج كلى تحقيق حاضر حاكى از آن است كه رابطه معنى بار

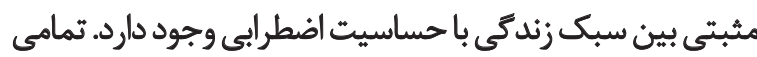

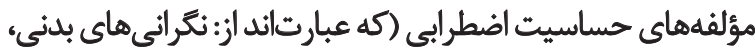

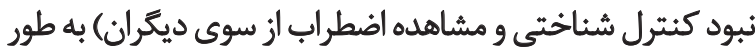

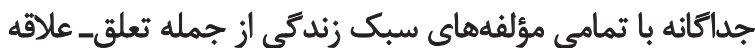

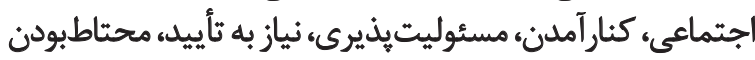

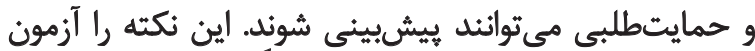

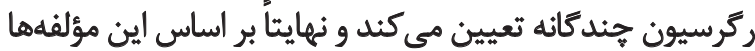

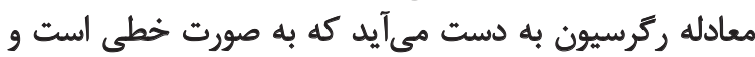

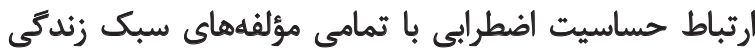

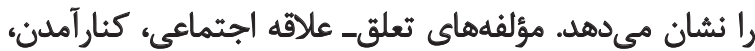

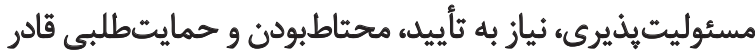

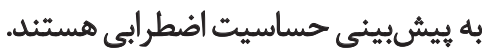

نتايج اين تحقيق با تحقيقات انجامشده در اين زمينه، همسو

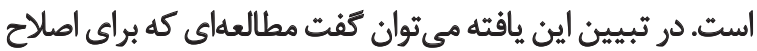

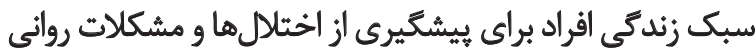

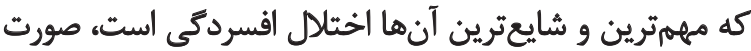


سبك زندگى قدرت بيشبينى عملكرد تحصيلى را ندارند. از

اين مطالعه حاصل باياننامه كارشناسىارشد نويسنده اول،

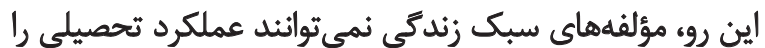

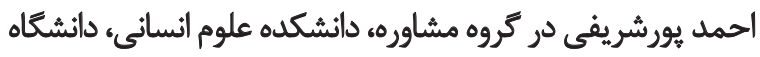
آزاد اسلامى، واحد ابهر است.

مشاركت نويسند كَان

مفهومسازى: اصغر جعفرى؛ تحقيق، بررسى، ويراستارى و نهايى سازى:احمد يورشريفى. تعارض منافع

بنابر اظهار نويسندكان، اين مقاله تعارض منافع ندارد.

سياسكّزَارى

از معاونت آموزشى دانشكاه علوم يزشكى قرئ مزوين و دانشجويان

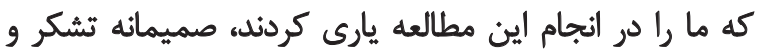

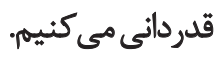

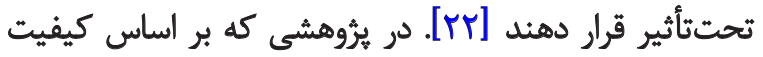

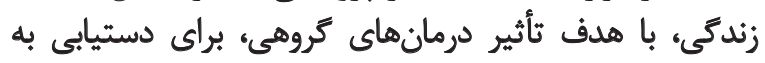

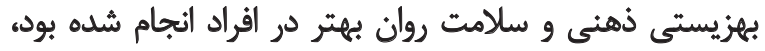

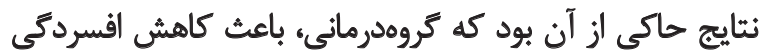

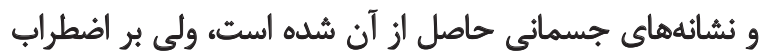

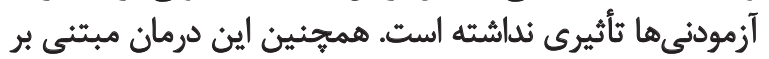

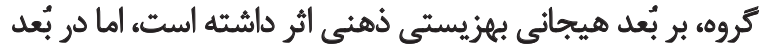

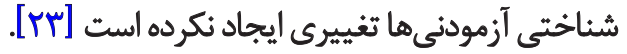

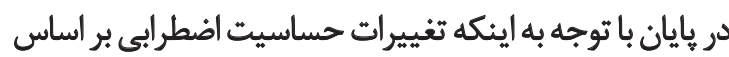

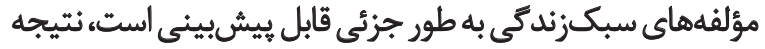

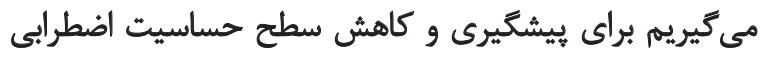

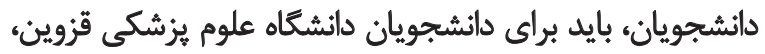

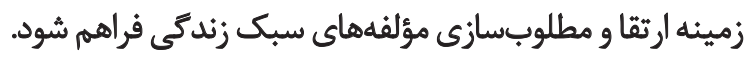
از آنجا كه بين مؤلفههاى سبك زندكى با حساسيت اضطرابى

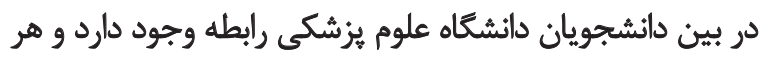

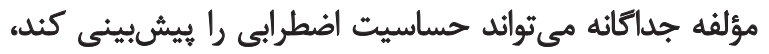

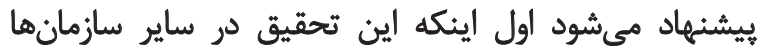

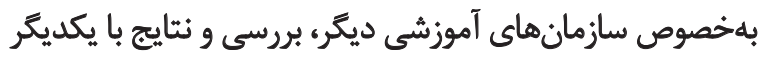

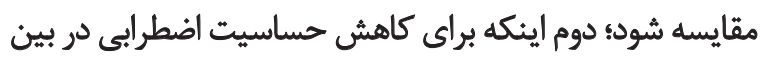

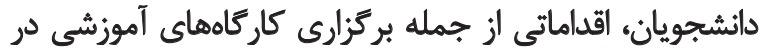

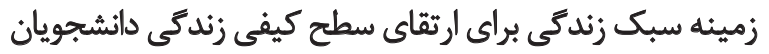

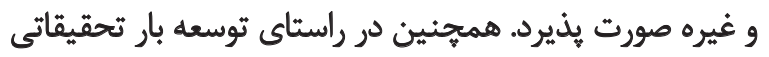

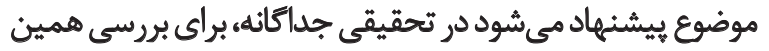

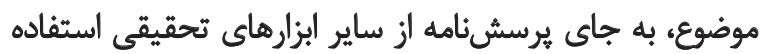

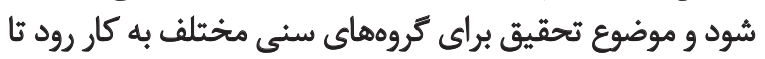
بتوان نتايج را با اطمينان بيشترى ارئي برائ داد.

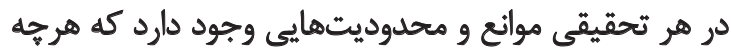

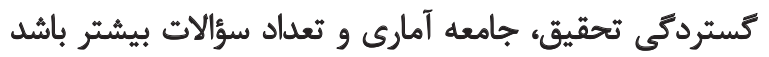

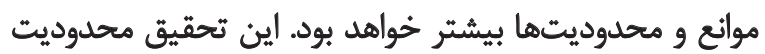

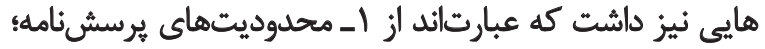

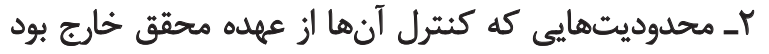

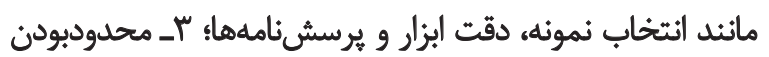

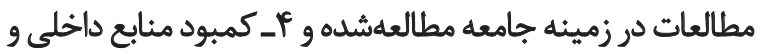
خارجى بهدليل جديدبودن موضوع مطالعهشده.

مالاحظاث اخخالقي

$$
\text { بيروى أز اصول اخلاق ثئوهش }
$$

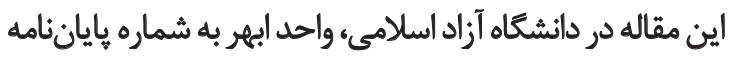

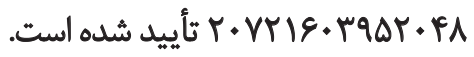




\section{References}

[1] Richeya JA, Schmidtc NB, Hofmannd SG, Timpano KR. Temporal and structural dynamics of anxiety sensitivity in predicting fearful responding to a 35\% CO2 challenge. J Anxiety Disord. 2010; 24(4):423-32. [DOI:10.1016/j.janxdis.2010.02.007] [PMID]

[2] Cox BJ, Enss MW, Freeman P, Walker JR. Anxiety sensitivity and major depression: Examination of affective state dependence. Behav Res Ther. 2010; 39 (11):1349-56. [DOI:10.1016/S00057967(00)00106-6]

[3] Golmohammadi K, Mantashloo S, Tavana Z. The role of metacognitive beliefs and anxiety sensitivity in prediction of social anxiety. J Mazandaran Uni Med Sci. 2016; 26(136):179-84. [In Persian]

[4] Abedi AA, Taher M, Jafari Sani B, Mojarrad A. The effect of attention control training on attention bias and anxiety sensitivity in students with social anxiety disorder. Q J Child Mental Health. 2017; 4(1):3-12. [In Persian]

[5] Mashhadi A, Gasempoor A, Akbari E, ElBeigi R, Hasanzadeh S. The role of anxiety sensitivity and emotion regulation in prediction of students social anxiety disorder. Knowl Res Appl Psychol. 2013; 14(2):89-99. [In Persian]

[6] Amani M, Shiri E, Valipoor M, Shiri V. The role of anxiety sensitivity and cognitive emotion regulation in the anxiety and depression. J Res Psychol Health. 2013; 7(1):29-39. [In Persian]

[7] Saedi Z, Farahbakhsh K. Relation among Quality of life, life style, academic performance with academic achievement in Isfahan University of Medical Sciences student's. Edu Strategies Med Sci. 2016; 9(3):176-85. [In Persian]

[8] Khajenoori B, Ruhani A, Hashemi S. Lifestyle and body image: Case study Shirazian Women. Iran J Cult Res. 2011; 4(1):79104. [In Persian]

[9] Skandari H, Gatezadeh A, Borjali A, Farrokhi N, Sohrabi F. Explanation of Depression Level Based On Lifestyle through the Mediation of Social Health and Quality Life. Int J Behav Sci. 2017; 10(3):145-50.

[10] Karami K, Rezadoust K, Abdizadeh S, Chebisheh F. Factors affected Health-oriented life style in students of Jundi Shapur University of Medical Sciences and Shahid Chamran University of Ahvaz. J Soc Dev. 2015; 10(1):97-126. [In Persian]

[11] Ahmadi B, Azmal M, Janani L, Bayatrizi M, Nooghani F. Healthy lifestyle and anthropometric measurements among employed women: The women's health initiative. J Payavard Salamat. 2017; 11(2):189-200. [In Persian]

[12] Sharifian F. Nasr A. Abedi L. Indicators of academic performance and evaluation of its implementation by faculty members. New Thought Edu. 2010; 1(1):9-33. [In Persian]

[13] Mohammadi D, Moslemi Z, Ghomi M. The relationship between critical thinking skills with creativity and academic achievement in students Qom University of Medical Sciences. Edu Strategies Med Sci. 2016; 9(1):79-89. [In Persian]
[14] Reiss S, Peterson RA, Gursky DM, McNally RJ. Anxiety sensitivity, anxiety frequency and the prediction of fearfulness. Behav ResTher. 1986; 24(1):1-8. [DOI:10.1016/0005-7967(86)90143-9]

[15] Naifeh JA, Tull MT, Gratz KL. Anxiety sensitivity, emotional avoidance, and PTSD symptom severity among crack/cocaine dependent patients in residential treatment. Cognit Ther Res. 2012; 36(3):247-57. [DOI:10.1007/s10608-010-9337-8] [PMID] [PMCID]

[16] Jafari A, Poyamanesh J, Gorbaniyan N, Kabiri M. The effectiveness of schema therapy on moderating early maladaptive schemas and reducing students' anxiety sensitivity. J Edu Sci. 2013; 6(24):79-91. [In Persian]

[17] Adler A. The individual psychology of Alfred Adler: A systematic presentation in selections from his writings. New York: Basic Book; 1956.

[18] Tagharobi Z. Predictors of academic performance of nursing graduates University of Medical Sciences. J Edu Strategies. 2011; 3(3):131-27. [In Persian]

[19] Norbala A, Damari B, Riyazi S. Lifestyle modifications in the prevention of common mental disorders, especially depressive disorders. Daneshvar Med. 2014; 22(113):1-12. [In Persian]

[20] Besharat MA, Baharamizade H, Fati L, Hasanabadi HR. Mediating role of metacognitive beliefs and anxiety sensitivity on the relationship between nauroticism with comorbidity of anxiety and depression. J Appl Psychol. 2015; 9(34): 7-23. [In Persian]

[21] Alimehdi M, Ehteshamzadeh P, Naderi F, Eftekharsadi Z, Pasha RA. Comparative study of the effectiveness of cognitive behavioral therapy and mindfulness based stress reduction on anxiety sensitivity and symptoms of generalized anxiety disorder. Middle East J Disabil Stu. 2017; 7:73. [In Persian]

[22] Prochaska JO, Norcross JC. Systems of psychotherapy: Atrans theoretical analysis [Y SeyedMohammadi, Persian trans]. Tehran: Ravan; 2010.

[23] Ghasemi N, Kajbaf MB, Rabiei M. The effectiveness of quality of life therapy (QOLT) on subjective well-being (SWB) and mental health. J Clin Psychol. 2011; 3(10):23-34. [In Persian] 
This Page Intentionally Left Blank 